\title{
Dual models of QCD and their implications
}

\author{
Deepak Bhatt ${ }^{1}$, H.C. Chandola ${ }^{1}$, Deependra Singh Rawat ${ }^{1}$ and H.C. Pandey ${ }^{2}$ \\ ${ }^{1}$ Department of Physics (UGC-Centre of Advanced Study), Kumaun University, Nainital-263001, India \\ ${ }^{2}$ Department of Physics, Birla Institute of Applied Sciences, Bhimtal-263136, India \\ Email: deepakbhattphysics@gmail.com
}

\begin{abstract}
Some of the dual formulations of QCD are reviewed and analyzed for their possible implications especially in non-perturbative sector of QCD at zero temperature. Starting from the Nambu-Mandelstam idea and the 't Hooft Abelian Projection technique, the effective formulations of QCD like the dual GinzburgLandau formulation and magnetic symmetry based dual QCD formulation have been analyzed in which dual magnetic potentials coupled with monopole field act as fundamental variables. The dual dynamics associated with these models is discussed in the quenched approximation and the analysis of symmetry breaking, flux tube configurations, confinement potential and nature of dual QCD vacuum is presented which establishes magnetic symmetry based dual QCD formulation as a more effective topological viable formulation for analysing the non-perturbative aspects of QCD.
\end{abstract}

Keywords: QCD, Abelian projection, Magnetic symmetry, monopoles.

\section{Introduction}

QCD, a non-Abelian gauge formulation of strong interactions is widely accepted as a best possible description of the hadronic world especially in its high energy sector [1]. However, due to the running nature of QCD coupling constant, several mystical features like color confinement, dynamical breaking of chiral symmetry, non-trivial topologies etc start popping-up in its low energy regime [2-4]. Among all of them, the color confinement is one of the outstanding and less known features that need to be resolved at the fundamental level. There are several models proposed to explore the physical dynamics associated with color confinement, among which Abelian dominance and monopole dominance are most leading candidates.

In the present study, we have made a comparative investigation of existing effective models like 't Hooft Abelian projection model, DGL model and magnetic symmetry based field decomposition model in the context of color confinement alongwith their viability at different level of their description.

\section{2. 't Hooft Abelian Projection}

To resolve the color confinement problem of QCD, Nambu,'t Hooft and Mandelstam put forward the idea of dual version of microscopic theory of superconductivity in which QCD vacuum is regarded as a dual superconductor. In this picture the color electric flux is expected to get confined through the dual Meissner effect as a result of color magnetic condensation in QCD vacuum. Although, this picture looks quite simple, the introduction of color monopoles was the major issue in the success as well as a 
subject of intense debate among physicists. The appearance of color monopoles in non-Abelian gauge theory was first proposed by 't Hooft, based on the Abelian gauge fixing that reduces $\mathrm{SU}\left(\mathrm{N}_{\mathrm{c}}\right)$ non-Abelian gauge theory to $\mathrm{U}(1)^{\mathrm{Nc}-1}$ Abelian gauge theory supplemented with color monopoles that may appear as hedgehog like configuration corresponding to the non-trivial homotopy class on non-Abelian manifold $\Pi_{2}\left\{\mathrm{SU}\left(\mathrm{N}_{\mathrm{c}}\right) / \mathrm{U}(1) \mathrm{Nc}-1\right\}=Z_{\infty}^{N_{c}-1}$. This hedgehog configuration in the maximal Abelian gauge manifest itself as a singular points that identifies color magnetic monopoles in terms of residual Abelian gauge symmetry. In this scheme, the Abelian gauge fixing is expressed through the diagonalization of a gauge dependent variable $\mathrm{M}(\mathrm{x})=\mathrm{T}_{\mathrm{a}} \mathrm{M}_{\mathrm{a}}(\mathrm{x})$ (where $\mathrm{T}_{\mathrm{a}}$ are the generators of the gauge group)[5]. The transformation corresponding to the diagonalization is,

$\mathrm{M}(\mathrm{x}) \mathrm{T} \mathrm{M}^{\prime}(\mathrm{x})=\Omega(\mathrm{x}) \mathrm{M}(\mathrm{x}) \Omega^{-1}(\mathrm{x})=\mathrm{M}_{\mathrm{d}}(\mathrm{x})$

where $\Omega(\mathrm{x})=\exp \left(\mathrm{iT}_{\mathrm{a}} \chi_{\mathrm{a}}(\mathrm{x})\right)$ is a gauge function in $\mathrm{SU}\left(\mathrm{N}_{\mathrm{c}}\right)$ color space. The gauge in which $\mathrm{M}(\mathrm{x})$ is diagnolized is called maximal Abelian gauge and depends on the choice of scalar field M. For $\mathrm{N}_{\mathrm{c}}=2$, the Abelian gauge is obtained by aligning $\mathrm{M}_{\mathrm{a}}(\mathrm{x})$ along the third direction in color space.

$\mathrm{M} \rightarrow \mathrm{M}_{\mathrm{a}} \mathrm{T}_{\mathrm{a}} \rightarrow \Omega \mathrm{M} \Omega^{\dagger}=\eta \mathrm{T}_{3}=\left(\begin{array}{cc}\eta & 0 \\ 0 & -\eta\end{array}\right)$

where $\eta=\sqrt{ }\left(\mathrm{M}_{1}{ }^{2}+\mathrm{M}_{2}{ }^{2}+\mathrm{M}_{3}{ }^{2}\right)$.

In this Abelian gauge the gauge field (gluon field) can be separated into regular and singular parts,

$\mathbf{A}=\mathbf{A}_{\mathbf{a}} \mathrm{T}_{\mathrm{a}}=\mathbf{A}_{\mathrm{a}}{ }^{\mathrm{R}} \mathrm{T}_{\mathrm{a}}-\mathrm{e}^{-1} \mathbf{n}_{\boldsymbol{\varphi}} \quad(1+\cos \theta / \mathrm{r} \sin \theta) \mathrm{T}_{3}$ (1)

The diagonal component of the gluon field acquires a singular form corresponding to the points where gauge fixing is ill defined. These points appears as topological defects that acts as a source of chromomagnetic field with associated magnetic charge $\mathrm{g}=-(4 \pi / \mathrm{e}) \mathrm{T}_{3}$. Thus in the vicinity of the points where Abelian gauge fixing is irrelevant, the Abelian part of the gauge field behaves as if a magnetically charge object having charge $(\mathrm{g}=-4 \pi / \mathrm{e})$ is already existing there.

Although in t'Hooft Abelian projection technique the color monopoles introduced in very well defined mathematical order however, from physics point of view it suffers from some serious drawbacks. In this technique the separation of Abelian part is gauge dependent which is inconsistent with the fact the gauge independency of all natural phenomena's. In addition to this such reduction of symmetry breaks color gauge invariance according to Schlieder's theorem which is another serious drawback. Further this formalism also does not tell us exactly the actual component responsible to bring confining features in the theory.

\section{The Dual Ginzburg-Landau formulation}

The DGL formulation is one of the effective tool to explain the confining properties of QCD vacuum. It is based on dual Higgs mechanism and can be deduced from QCD Lagrangian considering the fact of Abelian dominance and monopole condensation [6]. Monopoles are brought into dynamics via Abelian projection. In the quenched approximation (absence of quarks), we have the DGL Lagrangian as,

$£=-1 / 4\left(\partial_{\mu} \mathbf{B}_{v}-\partial_{v} \mathbf{B}_{\mu}\right)^{2}+\left[D_{\mu}, \chi\right]^{\dagger}\left[D^{\mu}, \chi\right]-\lambda\left(\chi^{\dagger} \chi-\right.$ $\left.\chi_{0}^{2}\right)^{2}$

Where $B_{\mu}$ is the dual gauge field, $\chi$ is the monopole field and $\mathrm{D}_{\mu}\left(=\partial_{\mu}+\operatorname{igT} . \mathbf{B}_{\mu}\right)$ is the dual covariant derivative and $\mathrm{g}$ is the dual 
gauge coupling constant $\chi_{0}$ is the vacuum expectation value of monopole field. As the monopole field $\chi$ approaches its vacuum expectation value $\chi_{0}$, which then acts as an order parameter will spontaneously break the dual gauge symmetry of the system and leads the QCD vacuum into color electric confined phase resulting from dual Meissner effect and monopole condensation. In this magnetically dominated phase, the color electric interquark potential becomes of linearly rising nature that confines the color isocharges. In view of the above description, the DGL theory of color superconductor seems to provide a suitable explanation of color confinement through magnetic condensation however, the DGL Lagrangian is constructed using the assumption Abelian dominance and monopole condensation but their origin is still lacking here as in the earlier proposal made by 't Hooft. Monopoles being non-static solutions are made explicit as reasonably localized particle states by gauge fixing (Abelian projection) conditions in the theory which reduces the symmetry of Lagrangian and breaks color gauge invariance [7]. Further the stability issue of the color electric flux also demanded serious concern over the validity of the formulation.

\section{Field decomposition formulation based on magnetic symmetry}

Another effective formulation to explore the mystic confining features of QCD is the magnetic symmetry based field decomposition higher-dimensional formulation of non-Abelian QCD. In this model the magnetic symmetry is introduced in the theory as an additional isometry of the internal space described by a Killing vector field and has Cartan's subgroup
$(\mathrm{H})$ of the gauge symmetry group $(\mathrm{G})$ as its little group. The gauge covariant magnetic symmetry condition which restricts and reduce the dynamical degrees of freedom keeping full gauge degrees of freedom intact [8-11], is then given as,

$$
\mathbf{D}_{\boldsymbol{\mu}} \widehat{m}=\left(\partial_{\mu}+\mathrm{g} \mathbf{W}_{\boldsymbol{\mu}} \times\right) \widehat{m}=0
$$

$\widehat{m}$ being a scalar multiplet belonging to the adjoint representation of the gauge group $G$ leads to an exact solution for the gauge group $\mathrm{SU}(2)$ and little group $\mathrm{U}(1)$ as

$$
\mathbf{W}_{\boldsymbol{\mu}}=\mathrm{A}_{\mu} \widehat{m}-\mathrm{g}^{-1}\left(\widehat{m} \times \partial_{\mu} \widehat{m}\right)
$$

with $\mathrm{A}_{\mu}=\widehat{m} . \mathbf{W}_{\boldsymbol{\mu}}$, unrestricted by magnetic symmetry is Abelian in nature while the second term is restricted by magnetic symmetry and of topological in origin, since the multiplet $\widehat{m}$ may be viewed to define the homotopy of the mapping $\Pi_{2}\left(\mathrm{~S}^{2}\right)$ as $\widehat{m}: S_{R}^{2} \rightarrow \mathrm{S}^{2}=\mathrm{SU}(2) / \mathrm{U}(1)$ which ensures the appearance of monopoles in the theory. The dual structure and dual dynamics exist between color isocharges and topological charges becomes more evident when we express the gauge fields in magnetic gauge $\left(\widehat{m} \stackrel{U}{\rightarrow} \xi_{3}=(0,0,1)^{\mathrm{T}}\right)$ as $\mathbf{W}_{\boldsymbol{\mu}} \stackrel{U}{\rightarrow}\left(\mathrm{A}_{\mu}+\mathrm{B}_{\mu}\right) \hat{\xi}_{3}$ and $\mathbf{G}_{\boldsymbol{\mu} \mathbf{v}}=\left[\mathbf{W}_{\mathbf{v}, \boldsymbol{\mu}}-\mathbf{W}_{\boldsymbol{\mu}, \boldsymbol{v}}+\mathrm{g} \mathbf{W}_{\boldsymbol{\mu}} \times \mathbf{W}_{\mathbf{v}}\right] \stackrel{U}{\rightarrow}$ $\left(\mathrm{F}_{\mu v}+B_{\mu v}^{(d)}\right) \hat{\xi}_{3}$. The corresponding Lagrangian with built in dual structure $\left(\partial_{\mu} G^{\mu \nu}=j^{v}, \partial_{\mu} G^{(d) \mu v}=\right.$ $\mathrm{k}^{v}$ ), in quenched approximation may then be expresses as

$$
\begin{aligned}
& \mathcal{L}_{d}^{(m)}=-\frac{1}{4} B_{\mu \nu}^{2}+\left|\left[\partial_{\mu}+i 4 \pi g^{-1} B_{\mu}^{(d)}\right] \phi\right|^{2}- \\
& \mathrm{V}\left(\phi^{*} \phi\right)
\end{aligned}
$$

$$
\begin{gathered}
\text { where } \mathrm{V}\left(\phi^{*} \phi\right)=24 \pi^{2} \mathrm{~g}^{-} \\
{ }^{4}\left[\phi_{0}^{2}+\left(\phi^{*} \phi\right)^{2}\left\{2 \ln \phi_{0}^{-2}\left(\phi^{*} \phi\right)-1\right\}\right]
\end{gathered}
$$

is the one loop effective Coleman Weinberg potential which is responsible for the dynamical 
breaking of magnetic symmetry is fixed by the requirement of ultraviolet finiteness and infrared instability of dual QCD Lagrangian. The dynamical breaking of magnetic symmetry through dual Meissner effect then leads to the monopole condensation in QCD vacuum and confines the color isocharges. Consequently the associated color electric flux then squeezed in the form of thin flux tubes between the color isocharges. The field equations associated with the Lagrangian (5) are,

$$
\begin{gathered}
\left(\partial_{\mu}-i 4 \pi g^{-1} B_{\mu}^{(d)}\right)\left(\partial^{\mu}+i 4 \pi g^{-1} B^{(d) \mu}\right) \phi+ \\
24 \pi^{2} g^{-4}\left(4 \phi^{*} \phi \ln \frac{\phi^{*} \phi}{\phi_{0}^{2}}\right) \phi=0 \\
\partial^{v} B_{\mu \nu}+i 4 \pi g^{-1}\left(\phi \partial_{\mu} \phi^{*}-\phi^{*} \partial_{\mu} \phi\right)- \\
32 \pi^{2} g^{-4} B_{\mu}^{(d)} \phi \phi^{*}=0
\end{gathered}
$$

The close resemblance of these field equations with relativistic G-L field equations and their static vortex line solutions governs the flux tube structure inside the QCD vacuum. These field equations under cylindrical symmetry $(\rho, \varphi, z)$ along with the field ansatz $B_{\varphi}^{(d)}=-B(\rho)$, $B_{\rho}^{(d)}=B_{z}^{(d)}=0=B_{0}^{(d)}, \phi=\chi(\rho) \exp (\operatorname{in} \varphi)$ leads to the color electric field $E_{m}(\rho)=-\frac{1}{\rho} \frac{d}{d \rho}[\rho B(\rho)]$ and reduce the field equations in the following form,

$$
\begin{aligned}
& \frac{d}{d \rho}\left[\frac{1}{\rho} \frac{d}{d \rho}(\rho B(\rho))\right]-\frac{8 \pi}{g}\left[\frac{n}{\rho}+\frac{4 \pi}{g} B(\rho)\right] \chi^{2}(\rho)=0 \\
& \frac{1}{\rho} \frac{d}{d \rho}\left(\rho \frac{d \chi}{d \rho}\right)-\left[\left(\frac{n}{\rho}+\frac{4 \pi}{g} B(\rho)\right)^{2}+\frac{24 \pi^{2}}{g^{4}}\left(4 \chi^{2} \ln \frac{\chi^{2}(\rho)}{\phi_{0}^{4}}\right)\right] \chi(\rho)=0
\end{aligned}
$$

The asymptotic solution for $\mathrm{B}(\rho)$ in suitable boundary conditions [9-11] ensures the formation of color flux tubes, is given by

$\mathrm{B}(\rho)=-\frac{n g}{4 \pi \rho}[1+\mathrm{F}(\rho)]$ and $\mathrm{F}(\rho) \stackrel{\rho \rightarrow \infty}{\longrightarrow} \mathrm{C} \rho^{1 / 2} \exp (-$ $\left.\mathrm{m}_{\mathrm{B}} \rho\right)(8)$

Where $C$ is a constant and $m_{B}\left(=4 \pi g^{-1} \sqrt{2} \phi_{0}\right)$ is the glueball mass. Thus in nutshell the dynamical breaking of magnetic symmetry in QCD vacuum leads to the dual Meissner effect and sets two characteristic mass scales $m_{B}$ and $\mathrm{m}_{\phi}$. The glueball mass $\mathrm{m}_{\mathrm{B}}$ (which is defined as the vector mode) determines the magnitude of dual Meissner effect. The inverse of penetration depth $\lambda^{(\mathrm{D})}$ determines this mass. The other scale which is characterized by the scalar mode $\mathrm{m}_{\phi}$ corresponds to the threshold energy to excite the monopole in dual QCD vacuum. The inverse of this mass $\left(m_{\phi}^{-1}\right)$ leads to the coherence length $\xi^{(\mathrm{D})}$. The ratio of these two length scale determines the nature of dual QCD vacuum by evaluating associated DGL parameter, $K_{Q C D}^{(D)}=\frac{\lambda_{Q C D}^{(D)}}{\xi_{Q C D}^{(D)}}$ which for the infrared sector of QCD ( $\left.\alpha_{\mathrm{s}}=0.22\right)$ leads to the type-II superconducting behavior $(K>1)$ with multiflux tube structure of QCD vacuum. Thus, the magnetic symmetry based dual formulation nicely incorporates all the relevant degrees of freedom to explain the confining behavior of QCD vacuum by bringing duality at the fundamental level.

Besides proving Abelian dominance and magnetic condensation simultaneously, in this field decomposition formulation the gauge fixing is actually done at the level of magnetic symmetry itself, which reduces the principle fibre bundle to a subbundle supplemented by 
monopoles. It further splits the Abelian part in the chromoelectric and chromomagnetic components and clearly specifies one that is truly responsible for confinement in the theory which is quite difficult in earlier formulations. Monopole as global aspects of the gauge group here manifests itself at the level of particle interactions. For dual QCD formulation, being an additional isometry, the introduction of magnetic symmetry doesn't disturb the gauge symmetry of Lagrangian and color gauge invariance remains intact. This allows one to choose a gauge invariant, parity conserving monopole background and provides stable monopole condensation necessary for confinement as shown in recent Lattice QCD simulations [12-15]. Keeping in view the above advantages, topologically viable dual QCD formulation emerges as a more effective formulation for analyzing non- perturbative aspects of QCD. The further comprehensive investigation in this direction will be dealt in our forthcoming communications.

\section{Acknowledgements}

The authors are thankful to the organizing committee of APSLS-2018 for their motivation and support. The author (DSR) is also thankful to University Grants Commission, New Delhi for the financial assistance in the form of fellowship under UGC(BSR)-RFSMS scheme.

\section{References}

1. Gross D.J., Wilczek.F, Phys.Rev. Lett. 26, 3633 (1973).

2. Stack J.D., Wensely and Neiman S.D., Phys. Rev. D50, 3399 (1994).
3. Toki S., Kitahara S., Kiura S., Matsubara Y., Miyanmura O., Ohno S., and Suzuki T., Phys. Letts. B272, 326 (1991).

4. Nambu.Y , Phys Rev. D10, 4262 (1974); Mandelstam. S, Phys. Rep. C23, 245 (1976).

5. Hooft G t', Nucl. Phys. B190, 455 (1981).

6. Suganuma H., Sasaki S. and Toki H., Nucl. Phys. B435, 207 (1995).

Ichie H., Suganuma H., Toki H, Phys. Rev. D54, 3882 (1996).

7. Schlieder.S, Nuovo Cimento 63A , 137 (1981).

8. Cho.Y.M. Phys.Rev. D21, 1080 (1980).

9. Pandey H.C, Chandola H.C, Phys. Lett. B476, 193 (2000).

10. Chandola H.C., Yadav D., Pandey H.C., Dehnan H., Int. J. Mod. Phys. A20, 2743 (2005).

11. Chandola H.C. and Yadav D., Nucl. Phys. A829, 151 (2009).

12. Kronfeld A., .Sheirholz J., Nucl.Phys. B293, 461 (1987); Phys.Lett. B198, 516 (1987).

13. Brandstater F., Sheirholz G., Phys.Lett. B272, 319 (1999).

14. Kato S., Kondo K.., Murakami T., Shibata A., Shinohara T., Ito S., Phys. Lett. B632, 326 (2006).

15. Cundy N., Cho Y.M., Lee W., Leem J., Phys.Lett. B729, 192 (2014), Nucl. Phys. B895, 64 (2015). 